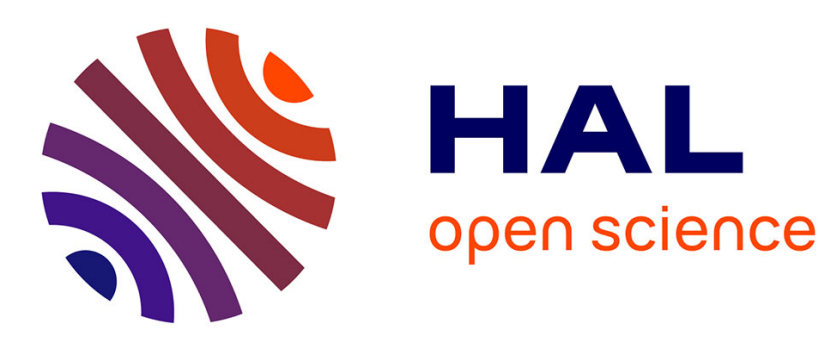

\title{
Empilement " boreal " pour le contrôle de la teneur en bore d'échantillons d'alliage bore-aluminium
}

\author{
J.-C. Carré, C. Letour
}

\section{To cite this version:}

J.-C. Carré, C. Letour. Empilement " boreal " pour le contrôle de la teneur en bore d'échantillons d'alliage bore-aluminium. Revue de Physique Appliquée, 1971, 6 (3), pp.267-272. 10.1051/rphysap:0197100603026700 . jpa-00243540

HAL Id: jpa-00243540

https://hal.science/jpa-00243540

Submitted on 1 Jan 1971

HAL is a multi-disciplinary open access archive for the deposit and dissemination of scientific research documents, whether they are published or not. The documents may come from teaching and research institutions in France or abroad, or from public or private research centers.
L'archive ouverte pluridisciplinaire HAL, est destinée au dépôt et à la diffusion de documents scientifiques de niveau recherche, publiés ou non, émanant des établissements d'enseignement et de recherche français ou étrangers, des laboratoires publics ou privés. 


\title{
REVUE DE PHYSIQUE APPLIQUÉE
}

\author{
Supplément au * Journal de Physique *
}

\section{EMPILEMENT «BOREAL » POUR LE CONTRÔLE DE LA TENEUR EN BORE D'ÉCHANTILLONS D'ALLIAGE BORE-ALUMINIUM}

\author{
J.-C. CARRÉ et C. LETOUR
}

Département des Etudes de Piles, Centre d'Etudes Nucléaires de Fontenay-aux-Roses

(Reçu le 19 novembre 1970)

Résumé. - Boreal est un empilement de graphite contenant une source de neutrons et des compteurs $\mathrm{BF}_{3}$, destiné à contrôler la teneur en bore ou en matériaux capturants d'alliage Al-B. Les mesures sont effectuées sur des plaquettes de $50 \times 50 \mathrm{~mm}^{2}$ par comparaison à une référence pour contrôler l'homogénéité et par rapport à des étalons pour déterminer la valeur absolue. Pour des plaques contenant $400 \times 10^{-6}$ partie de bore, la précision est de $2 \%$.

\begin{abstract}
Boreal is a small graphite array containing a neutron source. It is intended for checking the boron or absorber content of small slabs, for instance aluminium-boron alloy slabs.

The slabs, $50 \times 50 \mathrm{~mm}^{2}$, are compared to appropriate reference samples when checking the homogeneity and to standard samples for the determination of the absolute value. For slabs containing $400 \times 10^{-6}$ part of boron, the precision is about 2 per cent.
\end{abstract}

I. Introduction. - Les éléments combustibles à plaques des réacteurs de recherche du type " piscine » contiennent $\mathrm{du}$ poison consommable, en général $\mathrm{du}$ bore, dont il est nécessaire de connaître les teneurs. Cette détermination peut s'effectuer soit par analyse chimique, soit par la méthode d'oscillation [1], mais celles-ci sont en général longues et coûteuses.

Pour contrôler l'homogénéité et mesurer par rapport à des étalons la teneur en bore ou en matériaux capturants d'échantillons prélevés sur des plaques d'alliage Al-Bore, on a développé une méthode neutronique et réalisé un petit empilement «Boreal » dont le fonctionnement est automatique. On exposera ici, le principe utilisé et on donnera une description de l'ensemble de mesure ainsi que quelques résultats permettant d'évaluer ses performances.

II. Principe. - Lorsqu'on place un matériau absorbant dans un flux de neutrons thermique stationnaire créé par exemple par une source dans un bloc de graphite, on crée une perturbation de la densité neutronique qu'on peut facilement détecter avec un compteur.

Ce principe, déjà appliqué dans l'empilement Mireille [2] [3], pour la mesure de la pureté nucléaire du graphite, a été étendu au contrôle de l'homogénéité de la teneur en bore d'échantillons se présentant sous forme de plaquettes d'alliage ou de mélange aluminium-bore.

Sur la figure 1, on donne un schéma simplifié de l'ensemble Boreal. Dans un bloc de graphite, on produit un flux de neutrons thermique à l'aide d'une source Américium-Béryllium placée dans un boîtier de plexiglas pour thermaliser les neutrons. Un compteur

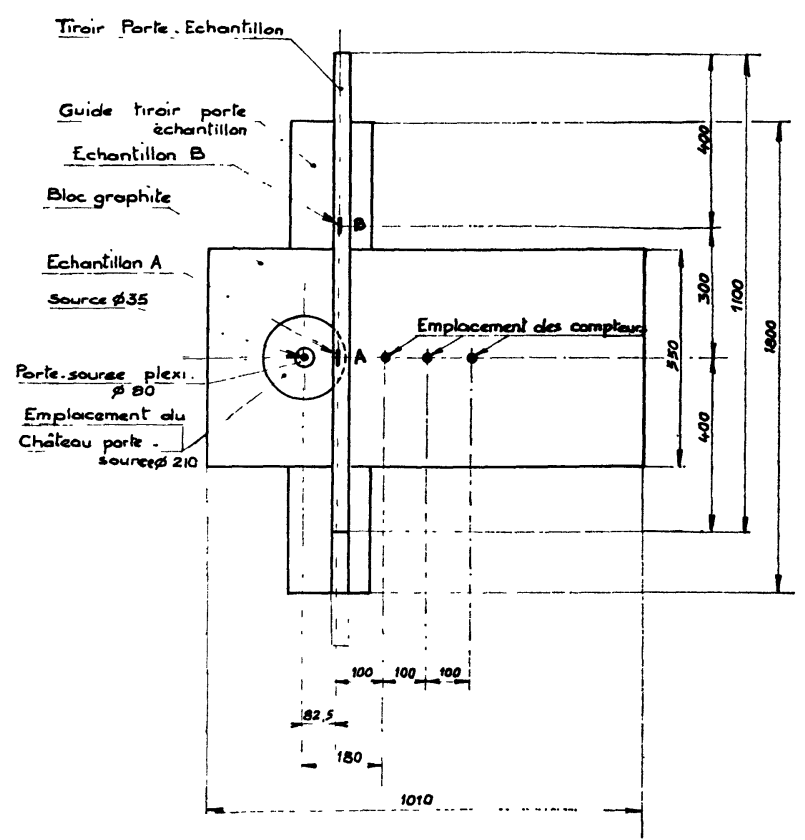

FIG. 1. - Schéma de principe du bloc de graphite (vue de dessus).

à $\mathrm{BF}_{3}$ permet de mesurer les variations de flux provoquées par l'interposition de l'échantillon à contrôler entre la source et le compteur.

La variation du taux de comptage $\Delta N$, provoquée par l'introduction d'un échantillon de surface de capture $S_{\mathrm{c}}$ et de diffusion $S_{\mathrm{d}}$ est donnée par :

$$
\Delta N=k_{1} S_{\mathrm{c}}+k_{2} S_{\mathrm{d}}
$$

dans laquelle $k_{1}$ et $k_{2}$ sont des constantes, lorsque les variations sont faibles. 
Pour des échantillons présentant la même diffusion, à une petite augmentation de $\Delta S_{\mathrm{c}}$ correspond une diminution du taux de comptage de :

$$
\Delta N=k_{1} \Delta S_{\mathrm{c}} .
$$

Cette variation est linéaire pour de petites variations de capture de l'absorbeur placé dans le bloc de graphite.

Sur le même principe, il est aussi possible d'évaluer la quantité de bore contenu dans l'alliage par comparaison à des étalons de captures connues.

III. Description. - L'ensemble Boreal se compose :

- d'un bloc de graphite qui comprend la source de neutrons et le compteur $\mathrm{BF}_{3}$,

- d'un porte-échantillon qui reçoit les échantillons à mesurer,

- de l'électronique de comptage associée au détecteur,

- d'un système de permutation automatique d'échantillons.

1) BLOC DE GRAPHITE. - Le bloc de graphite est constitué de briques de graphite de qualité nucléaire, ses dimensions extérieures sont de :

$$
550 \times 550 \times 1010 \mathrm{~mm}^{3}
$$

(cf. Fig. 1), il possède :

- un trou de $80 \mathrm{~mm}$ de diamètre dans lequel vient se loger le porte-source,

- une fente dans laquelle peut coulisser un porteéchantillon en graphite,

- trois trous borgnes espacés de $10 \mathrm{~cm}$ dans lesquels on peut introduire un compteur à $\mathrm{BF}_{3}$, de type $14 \mathrm{NE} 31$, de $25 \mathrm{~mm}$ de diamètre. Les deux autres trous inutilisés sont bouchés par un cylindre de graphite.

Le bloc de graphite est entouré de cadmium et recouvert d'une tôle de protection mécanique. Il est supporté par un assemblage de poutres qui lui sert de bâti.

La source d'Américium-Béryllium de 1,5 curie produisant $3,6 \times 10^{6} \mathrm{n} / \mathrm{s}$, est placée à l'intérieur d'un cylindre de « plexiglas » servant de ralentisseur.

2) PoRte-ÉChantillon. - Le porte-échantillon se présente sous la forme d'un tiroir en graphite dans lequel on place les plaquettes à contrôler de $50 \times 50 \times 4,75 \mathrm{~mm}^{3}$. On mesure la variation de taux de comptage de l'échantillon par rapport, soit à un témoin d'aluminium, soit à une plaquette d'alliage de référence : le porte-échantillon possède 2 emplacements A et B (cf. Fig. 1) qui permettent d'effectuer les deux mesures sans être obligé de changer de plaquettes au cours de ces deux séries de comptage. Il peut être déplacé par un système de commande, soit manuellement, soit automatiquement, des butées permettant de retrouver les mêmes positions d'échantillons par rapport à la source et au compteur.

Pour des échantillons de géométrie différente, on peut réaliser un autre type de cavité dans le tiroir, mais celui-ci conserve ses dimensions extérieures, longueur et symétrie, pour éviter les effets dus aux extrémités et à la distance entre la source et l'échantillon.

3) EleCtronique ASSOciéE. - L'électronique associée est classique. Elle se compose : d'un compteur à $\mathrm{BF}_{3}$ avec son alimentation, qui est suivi d'un préamplificateur relié par un câble coaxial à un ensemble de comptage (amplificateur, discriminateur, échelle) dont les résultats sont enregistrés sur une machine imprimante. Une base de temps et deux tiroirs de programmation régissent le cycle de mesures, et servent aussi à commander le système de permutation automatique.

L'opérateur peut choisir sur ces tiroirs les durées de comptages $T$ et du temps d'arrêt $t_{\mathrm{A}}$ entre deux mesures pendant lequel le contenu de l'échelle est imprimé sur bande et la permutation automatique d'échantillons éventuellement effectuée.

Ces différents éléments sont placés dans un châssistable sur lequel est posée l'imprimante.

4) Système de Permutation automatique. - Ce système comprend un vérin pneumatique dont le piston est solidaire du porte-échantillon ; il est commandé par une électro-vanne qui est excitée par des impulsions appropriées.

A la fin d'un comptage, sur A par exemple, d'un cycle de durée $T$ affichée sur l'un des tiroirs de programmation, une impulsion est envoyée sur un circuit électronique qui agit sur une électrovanne provoquant le déplacement du porte-échantillon par l'intermédiaire d'un vérin pneumatique. Ce changement est effectué en quelques secondes, temps inférieur à celui d'arrêt $t_{\mathrm{A}}$ affiché sur le deuxième tiroir de programmation. Un deuxième cycle de mesure peut commencer sur le deuxième échantillon $B$ et ainsi de suite.

Il est possible, en agissant sur un commutateur, de faire varier les temps $T$ et $t_{\mathrm{A}}$, de façon à les adapter à la nature des échantillons et à la précision demandée ; $T$ est en général pris égal à $1000 \mathrm{~s}$ et $t_{\mathrm{A}}$ à $5 \mathrm{~s}$.

IV. Etude expérimentale. - Une étude expérimentale a été entreprise pour déterminer les principales caractéristiques de l'empilement Boreal.

On a examiné l'influence sur le taux de comptage de la position de la source et de la plaquette, et de la diffusion des échantillons. On a aussi mesuré le coefficient reliant la variation $\Delta N$ à la capture apparente et le facteur caractérisant le spectre de neutrons où est effectuée la mesure, ce dernier terme permettant éventuellement de corriger les résultats dans le cas d'un échantillon présentant des résonances.

1) INFLuenCE DE LA POSITION DE LA SOURCE. - On a mesuré la variation de taux de comptage $\Delta N$ pour un échantillon absorbant de $50 \times 50 \mathrm{~mm}^{2}$ en fonction de la position verticale $Z$ de la source. Pour cette mesure, on utilise une plaquette de cadmium très absorbante pour en augmenter l'effet. On constate qu'un déplacement de $10 \mathrm{~mm}$ autour de la position habituelle $Z=0$ 
$\Delta N_{c}$ en $\mathrm{c} / \mathrm{s}$

400

300

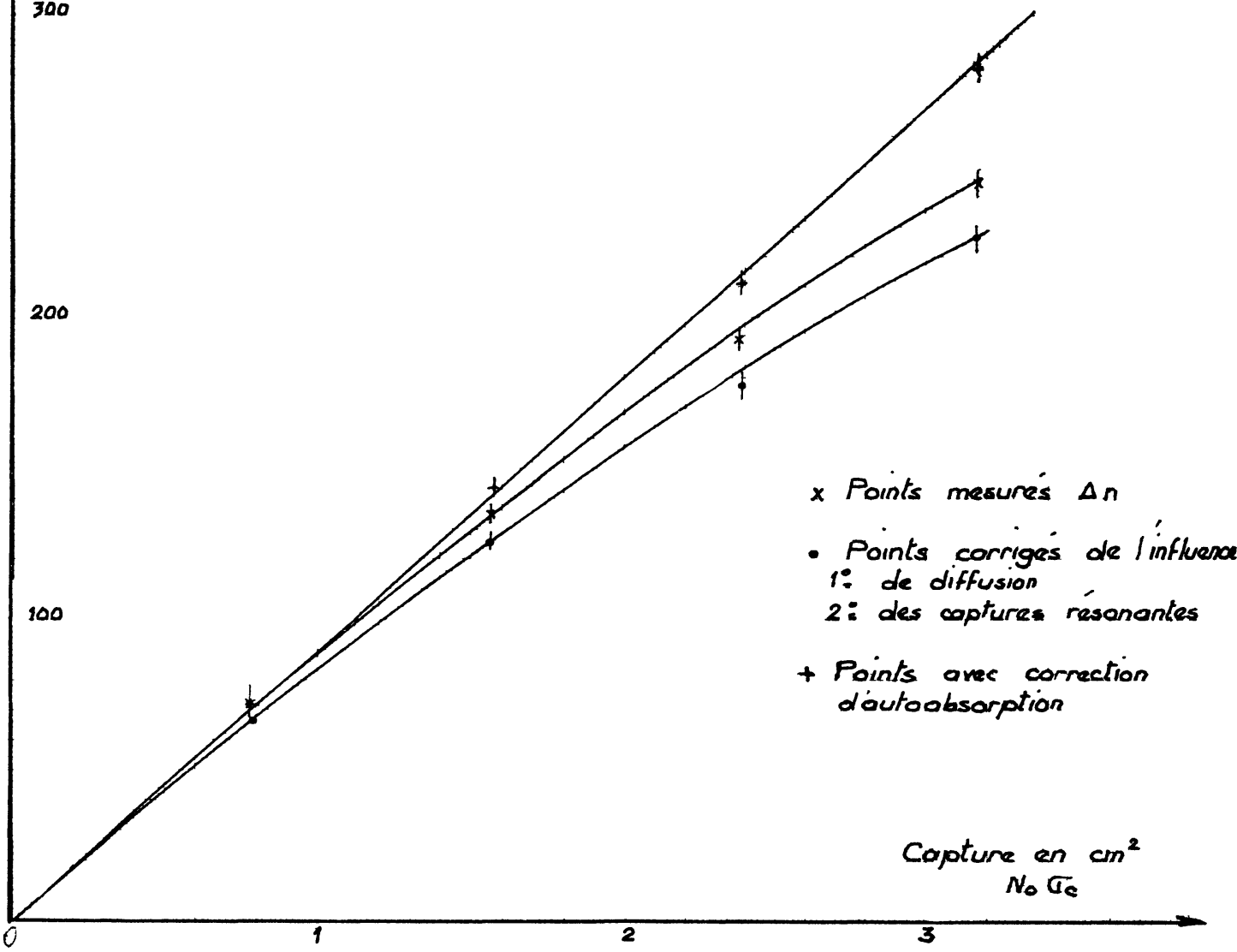

FIG. 2. - Courbe $\Delta N_{\mathrm{c}}=\mathrm{f}$ (capture du cuivre).

de la source, se traduit par une variation de taux de comptage inférieur à $1 \%$.

2) INFLUENCE DE LA POSITION DES ÉCHANTILLONS. On a imposé des déplacements $x$ de quelques centimètres au porte-échantillon contenant une plaquette absorbante de $50 \times 50 \mathrm{~mm}^{2}$. Expérimentalement, on a observé qu'une variation de position de $1 \mathrm{~mm}$ se manifeste par un $\delta \Delta N / N$ inférieur à $0,2 \%$. Pour n'introduire qu'une incertitude négligeable, on doit assurer une reproductibilité de position de l'échantillon dans le support mobile et de celui-ci dans l'empilement, meilleure que le millimètre.

3) Etalonnage en CAPture. - On sait que :

$$
\Delta N=k_{1} S_{\mathrm{c}}+k_{2} S_{\mathrm{d}}
$$

avec $S_{\mathrm{c}}$ et $S_{\mathrm{d}}$ surfaces de capture et de diffusion de l'échantillon.

Lorsqu'on utilise des échantillons de même nature la diffusion est identique pour chacun d'eux et la différence de taux de comptage est directement proportionnelle à $\Delta S_{\mathrm{c}}$.

Lorsqu'on effectue des mesures absolues les échantillons peuvent être de natures différentes, on a utilisé dans notre cas le cuivre comme étalon, la valeur des coefficients est alors :

$$
\begin{aligned}
& k_{1}=89 \pm 3 \mathrm{c} / \mathrm{s} \mathrm{mm}^{2} \\
& k_{2}=2,5 \pm 0,2 \mathrm{c} / \mathrm{s} \mathrm{mm}^{2}
\end{aligned}
$$

La relation (3) n'est exacte que pour de faibles absorptions, pour des échantillons plus capturants il se 
produit une autoabsorption et la relation n'est plus linéaire, on mesure donc une surface de capture apparente :

$$
\Delta S_{\mathrm{c}}^{\prime}=F \cdot \Delta S_{\mathrm{c}} .
$$

$F$ : coefficient d'autoabsorption.

Ce coefficient peut être soit calculé, soit mesuré si on possède une série d'étalons de teneurs absolues parfaitement connues.

Remarque. - Dans certains cas, le matériau à mesurer possède des résonances de capture (or, fer, cuivre), la capture réelle mesurée $\sigma_{\mathrm{c}}$ comprend alors la capture thermique $\sigma_{0}$ et la capture résonnante $\sigma_{\mathrm{r}}$. Il est commode de rapporter les valeurs à la capture thermique par une correction supplémentaire, qui fait intervenir l'intégrale de résonance du matériau $I$ et la composante épithermique du spectre $r$ :

$F \sigma_{0}=\sigma_{\mathrm{c}}-\sigma_{\mathrm{r}}=\sigma_{\mathrm{c}}-r \sqrt{\frac{4}{\pi}} F_{\mathrm{r}} I \quad(r=0,04)$.

On a représenté sur la figure 2 la courbe d'étalonnage donnant $\Delta N_{\mathrm{c}}$ en fonction de la surface de capture thermique réelle pour le cuivre et les différentes corrections de résonance, d'autoprotection $F_{\mathrm{r}}$, d'autoabsorption $F$ et de diffusion qui rendent la courbe linéaire.

Il est nécessaire d'effectuer un nouvel étalonnage si l'on change la forme et les dimensions des échantillons à contrôler. Celui qui est donné ici est relatif à des plaques de $50 \times 50 \mathrm{~mm}^{2}$.

V. Exemples de contrôle. - Sur l'ensemble Boreal deux types de contrôle peuvent être effectués, l'un concerne la détermination de la teneur en bore, l'autre la vérification de l'homogénéité d'une fabrication. Ces opérations peuvent se ramener à un seul type de mesure : variation du taux de comptage de l'échantillon par rapport, soit au témoin d'aluminium, soit à la plaquette de référence.

Avec le système de permutation automatique, on charge dans le porte-échantillon deux plaquettes $\mathrm{X}$ et $\mathrm{Y}$. Pendant des temps $T$ on mesure le taux de comptage avec $Y$ devant la source, puis avec $X$ et ainsi de suite. L'échantillon $Y$ sera pour la mesure de la teneur en bore le témoin $T_{\mathrm{c}}$ et pour le contrôle d'homogénéité la référence $\mathrm{R}$.

L'entrelacement des relevés élémentaires permet au besoin de corriger l'influence d'une éventuelle dérive de comptage et ainsi de contrôler la stabilité de l'ensemble.

1) Mesure de la teneur en Bore. - Pour effectuer cette détermination, on mesure successivement les taux de comptage de la plaquette témoin sans bore $T_{\mathrm{c}}$ et de l'échantillon $\mathrm{X}, \mathrm{La}$ différence $\Delta N_{\mathrm{x}}$ entre ces deux résultats est due à la capture apparente du bore contenu dans l'alliage.

Cette valeur $\Delta N_{\mathrm{x}}$ permet de déterminer la quantité de bore $m_{\mathrm{Bx}}$ contenu dans l'échantillon, à l'aide de la courbe d'étalonnage représentée sur la figure 3 , don- nant les $\Delta N_{\mathrm{x}}$ en c/s en fonction de $m_{\mathrm{Bx}}$ en mg de bore enrichi à $90 \%$ de ${ }^{10} \mathrm{~B}$. L'étalonnage est effectué avec le cuivre dont on exprime la capture en $\mathrm{mg}$ de bore équivalent.

Connaissant $m_{\mathrm{Bx}}$, il est aisé de calculer la teneur en bore de l'échantillon de masse $M_{\mathrm{x}} \mathrm{g}$ d'alliage soit :

$$
T_{\mathrm{x}}=\frac{m_{\mathrm{Bx}}}{M_{\mathrm{x}}}
$$

A titre d'exemple, examinons le cas d'un échantillon pour lequel on a trouvé un $\Delta N_{\mathrm{x}}=212 \mathrm{c} / \mathrm{s}$. Sur la courbe de la figure 3 , on détermine un $m_{\mathrm{Bx}}=14,2 \mathrm{mg}$. L'échantillon ayant une masse de $31,3 \mathrm{~g}$, on a :

$$
T_{\mathrm{x}}=\frac{14,2}{31,3 \times 10^{3}}=454 \times 10^{-6} \text { partie de bore } .
$$

On signalera qu'une différence de masse de 1 g d'AG3 entre l'échantillon et le témoin se traduit par une différence de taux de comptage de $0,54 \mathrm{c} / \mathrm{s}$; pour la plaquette de l'exemple, on aurait ainsi une différence de $0,3 \%$.

Pour que la différence de masse entre $T_{\mathrm{c}}$ et $\mathrm{X}$ introduise un effet négligeable elle doit être inférieure à $1 \mathrm{~g}$, sinon, il faut effectuer une correction.

2) Contrôle DE L'HOMOgÉNÉITÉ. - La différence $\Delta N_{\mathrm{x}}$ de taux de comptage entre l'échantillon $\mathrm{X}$ et la référence $\mathrm{R}$ sera portée sur la courbe d'étalonnage à partir du point correspondant à $R$, en tenant compte du signe de $\Delta N_{\mathrm{x}}$. On obtiendra ainsi la différence de masse de bore $\Delta m_{\mathrm{Bx}}$ par rapport à $\mathrm{R}$.

$\mathrm{Si}$ on a un grand nombre de plaquettes à mesurer, on pourra établir un abaque donnant directement $\Delta M_{\mathrm{Bx}}$ en fonction de $\Delta N_{\mathrm{x}}$.

Si les masses $M_{\mathrm{x}}$ de l'alliage de l'échantillon X et $M_{\mathrm{R}}$ de la référence $\mathrm{R}$ sont égales, on obtient aisément la différence de teneur :

$$
\Delta T_{\mathrm{x}}=T_{\mathrm{x}}-T_{\mathrm{r}}=\frac{\Delta m_{\mathrm{Bx}}}{M_{\mathrm{R}}} .
$$

Si les masses $M_{\mathrm{x}}$ et $M_{\mathrm{R}}$ présentent une différence, il faut appliquer une correction à $\Delta T_{\mathrm{x}}$.

On a trouvé pour une plaquette que l'on a comparée à l'échantillon de l'exemple précédent : $\Delta N_{\mathrm{x}}=20 \mathrm{c} / \mathrm{s}$ qui correspond à un $\Delta m_{\mathrm{Bx}}$ de $1,7 \mathrm{mg}$ de $\mathrm{B}$ soit $\Delta T_{\mathrm{x}}=54 \times 10^{-6}$ partie de bore. On obtient ainsi une teneur $T_{\mathrm{x}}$ de $400 \times 10^{-6}$ partie de bore.

VI. Précision. - La précision désirée sur l'évaluation de la teneur en bore ou sur le contrôle de l'homogénéité détermine le temps de mesure et le mode opératoire à adopter.

\section{Cette précision dépend :}

- de la statistique du taux de comptage, qui est fonction du temps de mesure,

- de la reproductibilité des mesures, qui dépend de la stabilité de la chaîne de détection et du positionnement des échantillons, 


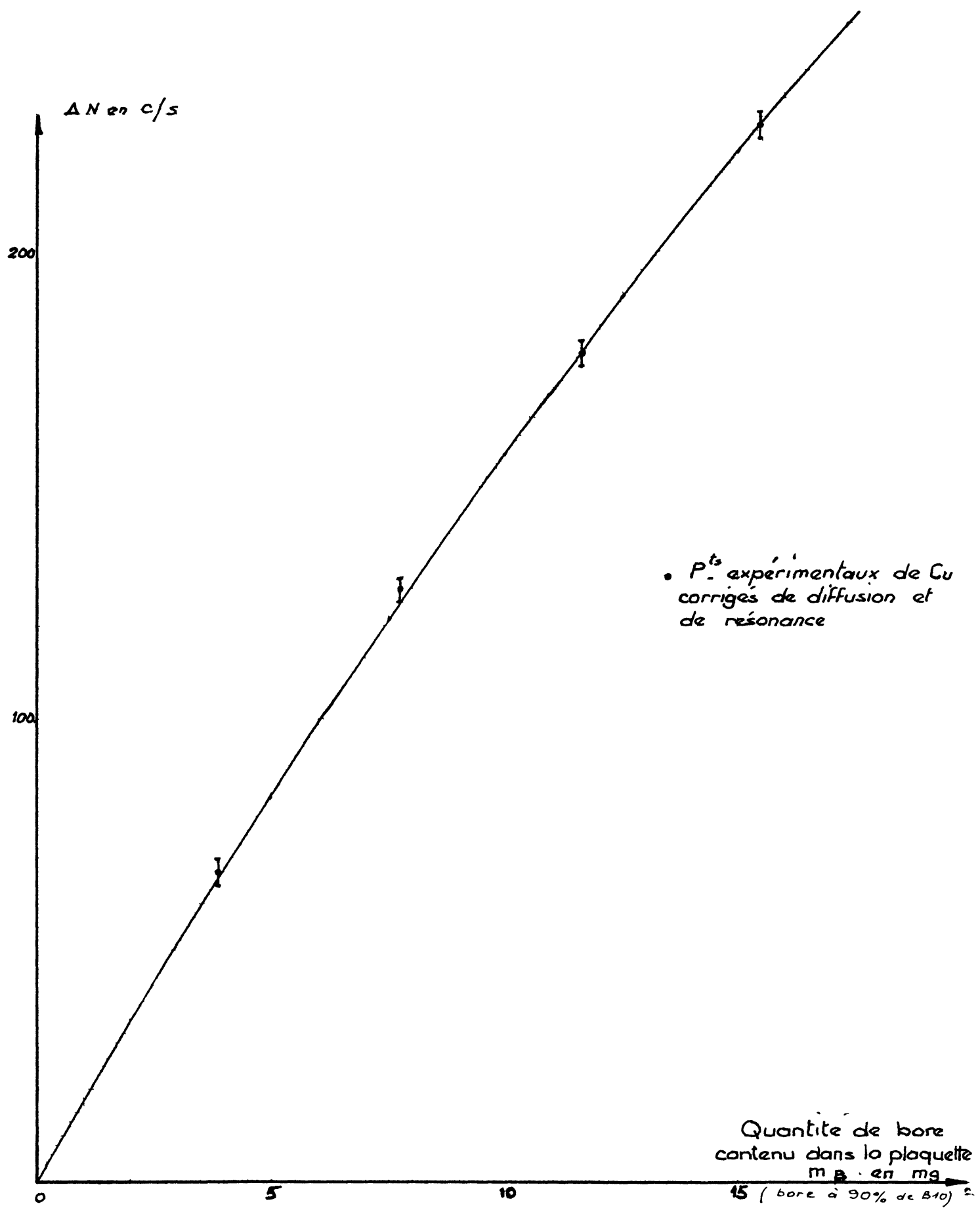

Fig. 3. - Mesure de la teneur en bore. Courbe d'étalonnage $\Delta N=\mathrm{f}\left(m_{\mathrm{B}}\right)$. Echantillon de $50 \times 50 \times 5 \mathrm{~mm}^{2}$.

- des différentes corrections (géométrie, différence de masse avec le témoin d'aluminium, ou la référence, diffusion).

1) Stabilité. - Une série de mesures avec un échantillon $\mathrm{X}$ et un témoin $T_{\mathrm{c}}$ effectuée sur une période d'une vingtaine de jours, a montré que l'écart quadratique calculé sur les $\Delta N_{\mathrm{c}}$, résultat de la différence des mesures entre $\mathrm{X}$ et $T_{\mathrm{c}}$, chacune d'une durée de $1000 \mathrm{~s}$, était identique à celui obtenu par une statistique directe sur les taux de comptage. Cette concordance montre la bonne stabilité de l'ensemble.
2) Précision sur une mesure. - La mesure de la teneur en bore, ou le contrôle de l'homogénéité, consistent à effectuer une différence entre le taux de comptage obtenu avec un témoin d'aluminium ou un échantillon de référence et celui obtenu avec l'échantillon à mesurer.

Pour un temps de $1000 \mathrm{~s}$, pour chacune des plaquettes, on obtient :

$\sigma= \pm 4600 \mathrm{c}$ ou ramené à 1 seconde $: 4,6 \mathrm{c} / \mathrm{s}$.

Cet écart quadratique moyen relatif à un temps de mesure de $1000 \mathrm{~s}$ peut s'exprimer en $\mathrm{mg}$ de bore. 
Par exemple, pour un échantillon contenant $14 \mathrm{mg}$ de bore, la courbe d'étalonnage de la figure 3 montre qu'une erreur de $\pm 4,6 \mathrm{c} / \mathrm{s}$ autour de $212 \mathrm{c} / \mathrm{s}$ se traduit par une incertitude de $\pm 0,35 \mathrm{mg}$ de bore. La masse d'alliage étant de $31 \mathrm{~g}$, on observe, pour ces conditions de mesures, un écart quadratique moyen de :

$$
\sigma^{\prime}=\frac{35 \times 10^{-2}}{31 \times 10^{3}}=11 \times 10^{-6} \text { partie de bore } .
$$

Pour un temps de $2000 \mathrm{~s}$ on aurait :

$$
\sigma=8 \times 10^{-6} \text { partie de bore. }
$$

3) Précision SUR Le CONTRôLe de L'HOMOgÉNéité. La précision sur le contrôle d'homogénéité d'une série de plaquettes, c'est-à-dire sur la mesure de la différence de teneur en bore par rapport à une référence $\mathrm{R}$, a pratiquement, comme valeur limite, l'écart quadratique moyen $\sigma$. Pour une mesure de $2000 \mathrm{~s}$, elle est égale ou inférieure à $8 \times 10^{-6}$ partie de bore.

4) Précision Sur la mesure de la teneur en bore. - Les erreurs entachant la mesure de la teneur en bore sont :

- l'erreur statistique qui peut être évaluée comme précédemment, pour un temps de mesure de $2000 \mathrm{~s}$, à $\pm 8 \times 10^{-6}$ partie de bore.

- l'erreur systématique provenant de l'imprécision sur l'étalonnage en capture.

Les erreurs provenant des corrections de masse d'aluminium et de variations de position peuvent être négligées.

L'imprécision sur l'étalonnage est due, dans le cas de l'emploi de plaquettes de cuivre :
- à la correction de diffusion et de résonance $(0,5 \%)$;

- à l'erreur entachant le $\sigma_{\mathrm{Cu}}(0,5 \%)$;

- à la statistique des points de mesure servant à tracer la courbe d'étalonnage (de 0,5 à $2 \%$ ), qui peut être améliorée en augmentant le temps de mesure.

L'avantage d'utiliser le cuivre comme étalon est dû à la possibilité de définir avec précision la surface de capture. Bien que par rapport au bore des corrections de diffusion et de résonance soient nécessaires, on peut atteindre sur l'étalonnage $\pm 1 \%$.

Dans ces conditions, la précision sur la teneur en bore des échantillons est suivant les cas de 2 à $3 \%$ soit de 8 à $12 \times 10^{-6}$ pour un échantillon en contenant $400 \times 10^{-6}$.

Sur les exemples cités précédemment, cette méthode a donné sur deux échantillons respectivement 454 et $400 \times 10^{-6}$ à comparer aux valeurs obtenues par analyses chimiques de 446 et $398 \times 10^{-6}$.

Ainsi la précision de mesure est de $10^{-5}$ partie de bore enrichi à $90 \%$ pour un temps de mesure de $2000 \mathrm{~s}$. Elle est comparable à celle des analyses chimiques.

VII. Conclusion. - Cette méthode utilisant un appareillage simple permet de mesurer la teneur en bore de plaques d'alliage Al-Bore avec une précision de $3 \%$, c'est-à-dire voisine de celle obtenue par analyse chimique, mais beaucoup plus rapide et moins coûteuse.

Il est possible d'envisager de mesurer avec le même ensemble la teneur en matériaux capturants d'échantillons de diverses natures, en effectuant un étalonnage approprié.

\section{Bibliographie}

[1] Breton (D.), Le contrôle des matériaux par la méthode d'oscillation à la pile de Châtillon, Rapport CEA, $n^{\circ} 400,1955$.

[2] Raievski (V.), Vidal (R.), Empilement pour le contrôle de l'absorption du graphite, Rapport CEA, n 839.

[3] RAIESKI (V.) et al., Empilement pour le contrôle des matériaux, 2e Conf. de Genève, 1958, 15, 1204, Vol. 13, 65-74.

[4] Carre (J.-C.), Vidal (R.), Mesures des sections efficaces et des intégrales de résonance par la méthode d'oscillation; Nuclear Data for reactors, Paris, 17-21 octobre 1966, Proceedings AIEA, 1967, I, 479-494. 